\title{
Measured and simulated transport of 1.9 MeV laser-accelerated proton bunches through an integrated test beam line at $1 \mathrm{~Hz}$
}

\author{
M. Nishiuchi, ${ }^{1, *}$ H. Sakaki, ${ }^{1}$ T. Hori, ${ }^{1}$ P. R. Bolton, ${ }^{1}$ K. Ogura, ${ }^{1}$ A. Sagisaka,,${ }^{1}$ A. Yogo, ${ }^{1}$ M. Mori, ${ }^{1}$ S. Orimo, ${ }^{1}$ \\ A. S. Pirozhkov, ${ }^{1}$ I. Daito, ${ }^{1}$ H. Kiriyama, ${ }^{1}$ H. Okada, ${ }^{1}$ S. Kanazawa, ${ }^{1}$ S. Kondo, ${ }^{1}$ T. Shimomura, ${ }^{1}$ M. Tanoue, ${ }^{1}$ Y. Nakai, ${ }^{1}$ \\ H. Sasao, ${ }^{1}$ D. Wakai, ${ }^{1}$ H. Daido, ${ }^{1}$ K. Kondo, ${ }^{1}$ H. Souda,${ }^{2}$ H. Tongu, ${ }^{2}$ A. Noda,${ }^{2}$ Y. Iseki,${ }^{3}$ T. Nagafuchi, ${ }^{3}$ K. Maeda, ${ }^{3}$ \\ K. Hanawa, ${ }^{3}$ T. Yoshiyuki, ${ }^{3}$ and T. Shirai ${ }^{4}$ \\ ${ }^{1}$ Advanced Photon Research Center and Photo Medical Research Center, Kansai Photon Science Institute, \\ Japan Atomic Energy Agency, 8-1 Umemi-dai, Kizugawa, Kyoto, 619-0215, Japan \\ ${ }^{2}$ Institute for Chemical Research, Kyoto University, Gokasho, Uji, Kyoto, 611-0011, Japan \\ ${ }^{3}$ New Technology Application Business Division, Toshiba Corporation, 1-1-1, Shibaura, Minato-ku, Tokyo, 105-8001, Japan \\ ${ }^{4}$ National Institute of Radiological Sciences, 4-9-1, Anagawa, Inage-ku Chiba-shi, 263-8555, Japan
}

(Received 11 March 2010; published 29 July 2010)

\begin{abstract}
A laser-driven repetition-rated $1.9 \mathrm{MeV}$ proton beam line composed of permanent quadrupole magnets (PMQs), a radio frequency (rf) phase rotation cavity, and a tunable monochromator is developed to evaluate and to test the simulation of laser-accelerated proton beam transport through an integrated system for the first time. In addition, the proton spectral modulation and focusing behavior of the rf phase rotation cavity device is monitored with input from a PMQ triplet. In the $1.9 \mathrm{MeV}$ region we observe very weak proton defocusing by the phase rotation cavity. The final transmitted bunch duration and transverse profile are well predicted by the PARMILA particle transport code. The transmitted proton beam duration of $6 \mathrm{~ns}$ corresponds to an energy spread near 5\% for which the transport efficiency is simulated to be $10 \%$. The predictive capability of PARMILA suggests that it can be useful in the design of future higher energy transport beam lines as part of an integrated laser-driven ion accelerator system.
\end{abstract}

DOI: 10.1103/PhysRevSTAB.13.071304

PACS numbers: 52.38.Kd, 52.50.Jm, 52.59.-f

\section{INTRODUCTION}

Development of high-power repetition-rated laser systems continues, following the first observation of proton acceleration by intense laser pulses irradiating thin solid targets $[1,2]$. As a result, laser-driven proton beam line development is still being widely examined both experimentally and theoretically. In the laser-plasma interaction, protons are accelerated from the rear (downstream) surface of the solid target by a strong quasistatic electric field of micron scale length with an acceleration gradient of order $\mathrm{MeV} / \mu \mathrm{m}$. This is the typical picture described with the target normal sheath acceleration model [1-3]. The divergent proton emission from the source can have very high single bunch charge and peak current $\left(>10^{12}\right.$ protons with duration near $1 \mathrm{ps}$ ) combined with an ultralow emittance [4-6].

Because of this compact and unique source size, laserdriven ion sources can attract many applications, which include injectors for conventional accelerators (hybrid systems) [7-9] and all-optical accelerators [10], and with the potential for development of compact facilities for laserdriven ion beam radiotherapy (L-IBRT) [11,12]. Most existing medical facilities that are based on conventional ion accelerators are typically large (in size and cost), thus limiting their number and ultimately access to ion beam

*nishiuchi.mamiko@jaea.go.jp radiotherapy. Consequently, there is strong interest in LIBRT and technologies that show promise for significantly reducing the size and cost of a medical ion accelerator.

However, in contrast to current medical ion accelerators, laser-driven sources can produce highly divergent proton emission with a very broad energy spread (typically $\sim 100 \%$ ), resulting in a potentially significant decrease in the number of protons delivered for therapy. These features represent serious challenges for development of laserdriven accelerator systems in general (for both all-optical and hybrid systems), where energy spread requirements can be at the percent level or lower.

Because of the large spatial divergence at the proton source, the initial collection and collimation optics are a critical part of the transport line for laser-accelerated ions. Permanent magnet quadrupole (PMQs) doublets and high field solenoids have been explored as candidates [9,13-15]. The current work described here presents new results using a PMQ triplet as the collection optic which is combined for the first time with an rf cavity and tunable monochromator located downstream. The investigation is conducted at 1.9 $\mathrm{MeV}$ and in the low space charge regime (as sketched in Fig. 1) as an initial step toward the development of an integrated laser-driven ion accelerator system (ILDIAS) [16].

The effect of the rf cavity on the proton bunch depends on the incident energy so it is important to assess its performance with a laser-driven source that has both spec- 


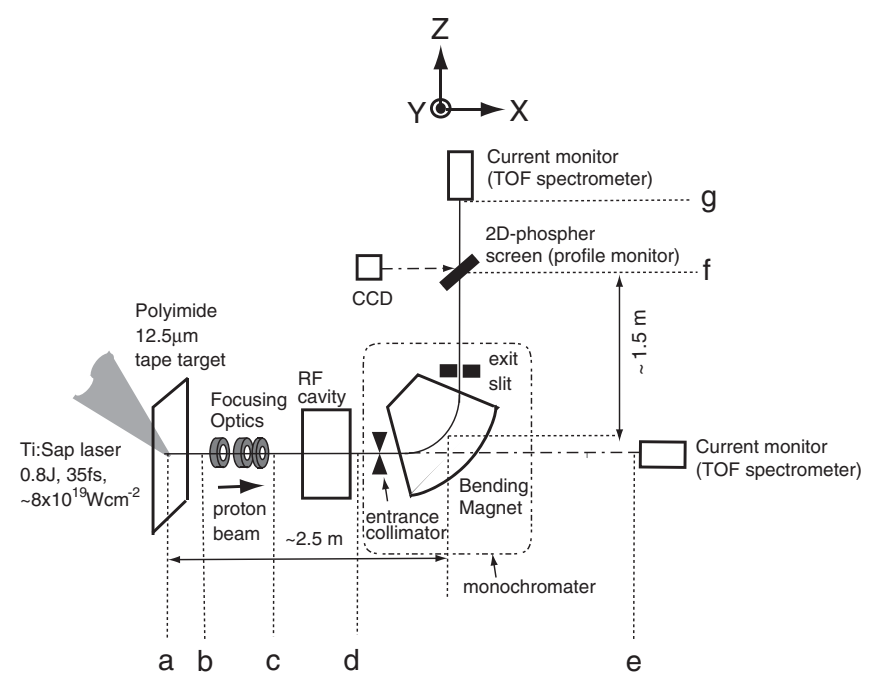

FIG. 1. Test transport line indicating planes referenced in the text. The axis $z$ is always along the proton beam direction.

trally broad features and a characteristically high divergence that is energy dependent. However, the divergence at the rf cavity input is significantly altered by the PMQ collection optics.

For advancing ILDIAS development, comparison with and validation of beam transport code is important. Because of the unique features of laser-driven sources, it is very important to compare transport measurements to the predictions of the transport codes over a wide range of source parameters, such as energy and bunch density. However, at this moment no such code has been tested for the laser-driven proton case because the research of the laser-driven proton accelerator is at the very early stages of development. For the current work we use the phase and radial motion in ion linear accelerator (PARMILA) code in this work [17], which has been already benchmarked with more conventional sources (which had significantly lower energy spread and divergence) and cross calibrated among five independent codes for the conventional accelerators [18]. The bunch duration and the transverse spatial distribution of the final transmitted quasimonoenergetic proton beam have been measured and compare favorably with the PARMILA code predictions. These transported bunch features are simulated based on an input spectrum and input transverse spatial profile that are measured upstream of the transport line.

\section{EXPERIMENTAL SETUP AND METHODOLOGY}

An intense, ultrashort laser pulse irradiates a thin solid foil target. The ensuing laser-plasma interaction accelerates protons from the target rear surface to $\mathrm{MeV}$ energies. As shown in Fig. 1, pulsed laser irradiation of a thin polyimide target generates accelerated protons as a single bunch for each laser pulse. We used the J-KAREN Ti: sapphire laser system at the Kansai Photon Science Institute of the Japan Atomic Energy Agency [19]. At a $1 \mathrm{~Hz}$ repetition rate (enabled by the polyimide tape target and its driver motion) $P$-polarized laser pulses with an energy near $630 \mathrm{~mJ}$, central wavelength of $800 \mathrm{~nm}$, and duration of $45 \mathrm{fs}$ (FWHM) irradiated a moving polyimide tape target of $12.5 \mu \mathrm{m}$ thickness at a $45^{\circ}$ incident angle. Peak irradiances up to $10^{19} \mathrm{~W} \mathrm{~cm}^{-2}$ have been achieved by focusing with an off-axis parabola (310.5 $\mathrm{mm}$ focal length) to spot sizes near $8 \times 4 \mu \mathrm{m}^{2}(\mathrm{FWHM}$, horizontal $\times$ vertical). Intensity contrast ratios typically at the $10^{10}$ level were obtained approximately 100 ps prior to the peak of the main pulse. With a fast Pockels cell the duration of the amplified spontaneous emission pedestal before the main pulse is reduced to $0.5 \mathrm{~ns}$.

The proton transport beam line is oriented parallel to the target normal. The PMQ triplet collects as many of the laser-accelerated protons as possible for transport downstream to the rf cavity device and the monochromator. The divergence of the proton source is centered on the beam axis. The acceptance half angle of the first PMQ is 7 degrees. The first, second, and third quadrupoles are placed at $(79.5 \pm 0.5) \mathrm{mm}$ from the target, $(128.5 \pm 0.5) \mathrm{mm}$ from the downstream end of the first one, and $(89.3 \pm 0.5) \mathrm{mm}$ from the downstream end of the second one as shown between planes "b" and "c" in Fig. 1. The magnetic field strengths (and magnet lengths) are $55 \mathrm{~T} / \mathrm{m}(50 \mathrm{~mm})$, $40 \mathrm{~T} / \mathrm{m}(50 \mathrm{~mm})$, and $60 \mathrm{~T} / \mathrm{m}(20 \mathrm{~mm})$, respectively.

Immediately downstream of the PMQ triplet is an rf cavity [20] that establishes phase space rotation in the proton bunch where the timing of the $80 \mathrm{MHz}$ rf is synchronized to that of the laser pulse with a $45^{\circ}$ phase offset. The rf cavity is composed of a quarter-wavelength resonator with two gaps. The gap size is $20 \mathrm{~mm}$ with an rf voltage amplitude of $115 \mathrm{kV}$. Downstream of the rf phase rotation cavity, there is the tunable proton monochromator which consists of an entrance collimator $(2 \mathrm{~cm}$ diameter), a tunable $90^{\circ}$ bending electromagnet, and an exit slit $(2 \mathrm{~cm}$ width). The field strength used for a $1.9 \mathrm{MeV}$ proton beam is 0.53 Tesla with a bending radius (gap height) of $400 \mathrm{~mm}$ $(65 \mathrm{~mm})$. It also serves as a final stage to separate protons from comoving residual electrons. Time-of-flight (TOF) detection of the bunch transmitted through the monochromator provides measurement of the final bunch duration and can detect significant spectral modulations if any are imposed by the rf cavity. The following experimental measurements of a proton bunch are made at four locations along the transport line as specified by planes indicated in Fig. 1: (i) source transverse spatial profiles at plane "b" using track detection in CR-39 film; (ii) time-of-flight (TOF) source spectra at plane "e"; (iii) transmitted transverse spatial profiles at plane " $\mathrm{f}$ " using a thin luminescent monitor; and (iv) transmitted TOF bunch durations at plane "g".

In plane "b", located $80 \mathrm{~mm}$ downstream of the source and in front of the first PMQ, nuclear track detection by 
CR-39 film is used with range filtering to determine energy-dependent transverse spatial bunch profiles typical of the bunch source. Following a 20 min etching process (in a 6 normality solution of $\mathrm{KOH}$ at $70^{\circ} \mathrm{C}$ ), the etched pits in the CR-39 that are attributed to impinging protons can be seen as whiter regions on the film. Because CR-39 has larger area and is located much closer to the source, the CR-39 film subtends a significantly larger detection solid angle than the TOF unit. The source proton spectra in plane "e" are measured $2.4 \mathrm{~m}$ downstream of the source by an absolutely calibrated TOF spectrometer [21]. For the source spectral measurements all transport line components are removed.

An insertable luminescent single bunch profile monitor (LSBPM) is used to measure the transmitted transverse bunch profile in plane "f" of Fig. 1. We plan to provide the details of this prompt profile monitor in a separate publication. By imaging proton-induced light emission onto a charge coupled device camera, we obtain prompt single bunch profiles at the $1 \mathrm{~Hz}$ repetition rate. The profile imaging system is capable of operation at a CCD limited repetition rate near $40 \mathrm{~Hz}$. It is noteworthy that the LSBPM diagnostics in plane "f" and the TOF spectrometry in plane "g" are insertable devices with prompt, repetitionrated single bunch detection capability.

The PARMILA code is a well-known particle code used to simulate the transport and acceleration of particles in accelerators $[17,18]$, but it has not been benchmarked for the laser-driven case. In our simulation, the rf field dynamics in the phase rotation device are computed with the electromagnetic solver, SUPERFISH (version 7.17), that creates static field maps. These maps enable determination of an energy-dependent transit time factor by the postprocessor of SUPERFISH, SF7 and this is an input to PARMILA. In contrast to the previous work $[13,22]$, the effect of space charge is evaluated by comparing the PARMILA simulation results with and without space charge calculations. This is done by running either the SCHEFF (2D) or PICNIC (3D) routine within PARMILA. PICNIC is included on account of the asymmetrical PMQ focusing [17]. We found that both SCHEFF and PICNIC give the same results. The number of meshes used in the space charge calculation is initially set to 80 radially [80 horizontally $(x)$ and vertically $(y)$ ] for SCHEFF (for PICNIC) and 200 in the longitudinal directions for both SCHEFF and PICNIC. The input file for PARMILA is created by using the initial beam generator [16] using the observed proton source parameters, which will be explained later in the text. We use $5 \times 10^{6}$ particles emitted simultaneously from the source where the energy distribution and momentum direction are set to match the observed results shown in the Fig. 2(a). That is, the particles are emitted with a Gaussian spatial distribution with a threefold standard deviation of 10 degrees. Peak current (at input plane to the first PMQ) was varied 0 to $1 \mathrm{~A}$. Simulation results were relatively insensitive to transverse emittance in the broad range that we tested, $0.001 \pi \mathrm{mm} \mathrm{mrad}$ to $0.1 \pi \mathrm{mm}$ mrad. An unnormalized transverse emittance of $0.01 \pi \mathrm{mm}$ mrad is therefore chosen for the PARMILA simulations.

\section{RESULTS AND DISCUSSION}

A typical energy spectrum of protons emitted from the tape source can be seen in Fig. 2(a). Also the typical transverse profile image, seen in Fig. 2(b), was acquired with a $40 \mu \mathrm{m} \mathrm{Al}$ range filter upstream for detection of protons in the energy range 1.9 to $2.2 \mathrm{MeV}$. It should be noted that the large divergence is mainly attributed to the electrostatic field distribution [23]. Based on this profile the emitted proton beam diameter (FWHM) is determined to be $(14 \pm 1) \mathrm{mm}$ which is consistent with a half-angle (a)

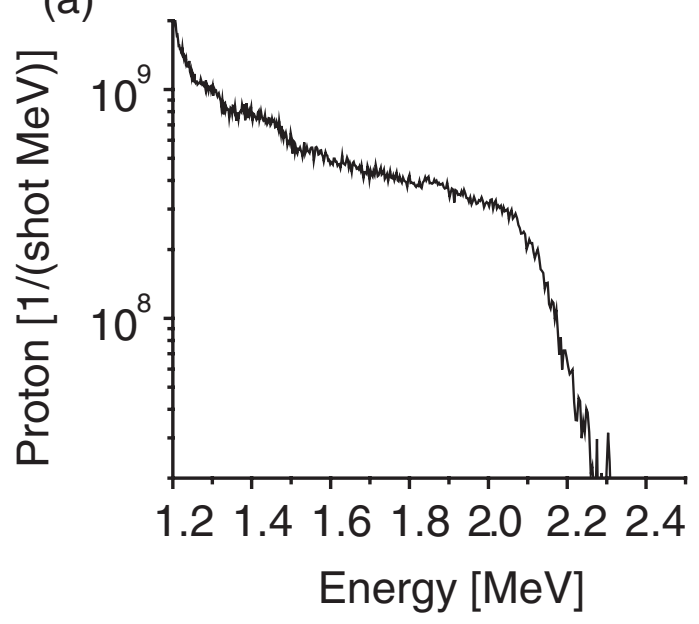

(b)

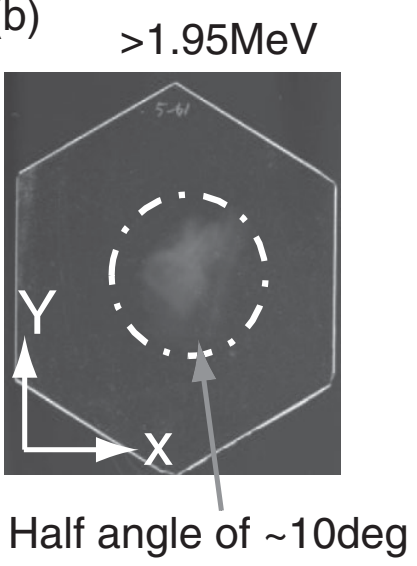

FIG. 2. (a) Typical time-of-flight proton energy spectrum from the polyimide tape source (laser target). (b) Typical transverse spatial profile of a proton bunch within the energy range of $1.9 \mathrm{MeV}<\mathrm{E}<2.2 \mathrm{MeV}$, as measured with the CR-39 nuclear track detector. The $x$ and $y$ axes in the figure correspond to those in Fig. 1 . 
a)

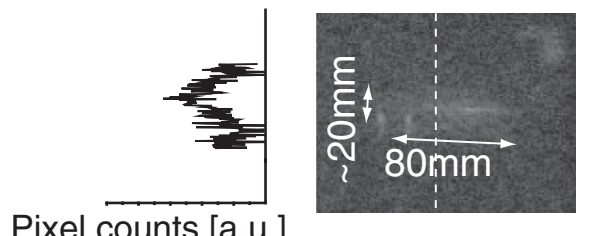

Pixel counts [a.u.]

0
28.32
56.64
84.96
113.3
145.0

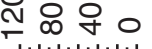

용ㅇㅇ c)

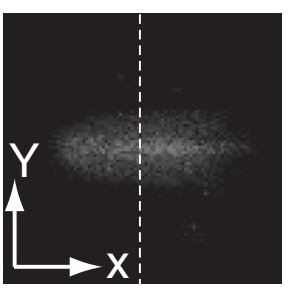

b)

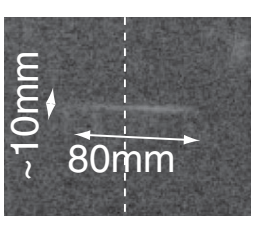

d)

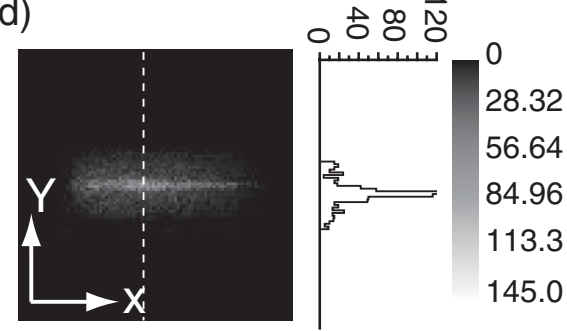

FIG. 3. Transverse bunch profiles without slit obtained with the LSBPM unit when rf is on (a) and off (b) at plane " $\mathrm{f}$ ". Those predicted by the PARMILA simulation when $\mathrm{rf}$ is on (c) and off (d) on the same scale. The line graphs beside the panels show the vertical projections of each image. The $x$ and $y$ axes in the figure correspond to those in Fig. 1.

divergence of $(10 \pm 0.5)$ degrees. This exceeds the halfacceptance angle of the first PMQ. Combining the TOF spectrometry results with angular scaling from CR-39 data, we determine that a single laser pulse generates more than $10^{11}$ protons at the polyimide tape source over the full spectrum and $(5.0 \pm 0.9) \times 10^{7}$ protons within the $(1.9 \pm$ $0.05) \mathrm{MeV}$ energy interval.

Assuming an initial bunch duration be near $1 \mathrm{ps}$, this represents a source peak current of order $320 \mathrm{~A}$ for the full spectrum and $8 \mathrm{~A}$ for the $5 \%$ energy bin at $1.9 \mathrm{MeV}$. Taking into account bunch duration increase associated with the large energy spread, the peak current incident on the first PMQ is reduced to about 0.14 A for the full spectrum (simulated bunch duration of $\sim 3 \mathrm{~ns}$ ) and to about $52 \mathrm{~mA}$ for the 5\% energy bin at $1.9 \mathrm{MeV}$ (simulated bunch duration of $0.1 \mathrm{~ns}$ ). Based on the PARMILA simulation, the bunch densities at this position are only $5 \times 10^{7} \mathrm{~cm}^{-3}$ for the full spectrum and $1.3 \times 10^{6} \mathrm{~cm}^{-3}$ for the $5 \%$ bin at $1.9 \mathrm{MeV}$. This latter value is the energy spectrum of the proton bunch that is transmitted through the beam line. It is more than an order of magnitude lower than a threshold value for significant emittance growth than we estimated from the reported work of Antici et al. [24].

In order to reproduce the spatial distribution shown in Fig. 2, the proton beam current at a source set in the PARMILA simulation should be $\sim 5 \mathrm{~mA}$. At this current level, the spatial distribution as well as the arrival time information at the end of the beam line are well reproduced. However, the current level is far below the estimated peak current at a source. One of the possible explanations for this may be the strong charge neutralization effect caused by the vacuum level of $10^{-6}$ Torr in our experiment [25]. Another factor which supports the strong charge neutralization may be the existence of the comoving electrons intrinsically generated from the target, which is a peculiar characteristic of the laser-driven protons [26]. Further investigation with respect to this issue should be carried out.

Given the typical broadband nature of laser-driven sources and the energy-dependent spatial divergence after passing through the PMQ focusing optics, it is important to characterize the energy gain (loss) and focusing (defocusing) performance of the rf cavity at various energies. For this work, at the nominal $1.9 \mathrm{MeV}$ proton energy, the cavity device is operated in an energy loss, defocusing mode with an incident peak current of $6 \mathrm{~mA}$ where the charge density of $2.5 \times 10^{6} \mathrm{~cm}^{-3}$ is very low (as with other current and density estimates, these values are estimated by scaling the PARMILA simulation to the measured charge level near the source). We estimate the defocusing

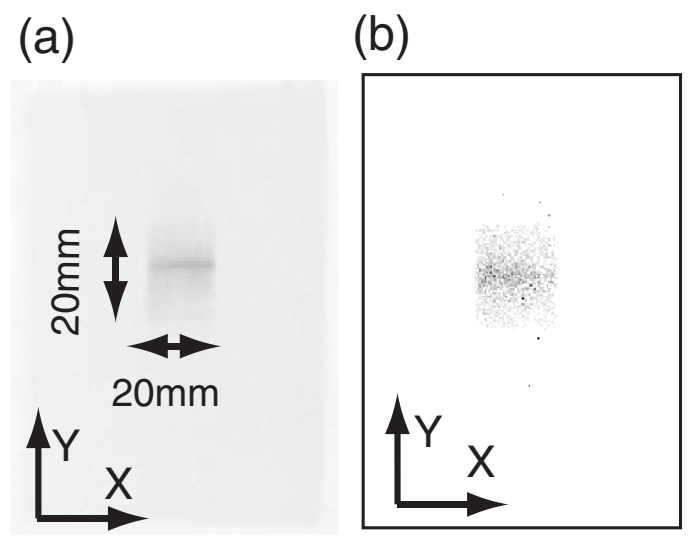

FIG. 4. (Color) (a) Observed beam pattern with reinstalled exit slit of the monochromator at plane " $\mathrm{f}$ " whose baseline spot size is $\sim 20$ (horizontal) $\times 20$ (vertical) $\mathrm{mm}$. (b) PARMILA simulated beam pattern at the same location as for (a) with the same scale. The $x$ and $y$ axes in both panels correspond to those in Fig. 1 . 
(a)

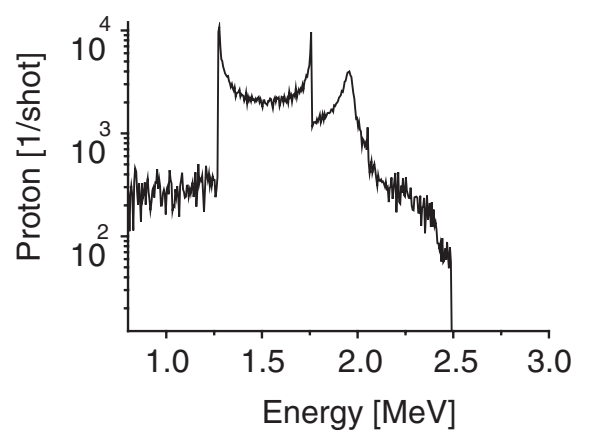

(b)

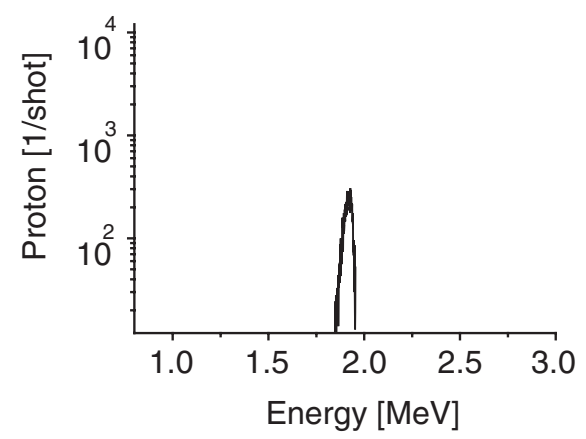

FIG. 5. (a) A PARMILA simulation of the modulated spectrum exiting the rf phase rotation cavity and subsequently entering the monochromator. (b) Simulated transmitted proton bunch spectrum at plane " $f$ " in Fig. 1.

strength to be in the range of $10^{-4}$ to $10^{-3} \mathrm{~m}^{-1}$ [22]. Furthermore, transverse bunch profiles obtained with the LSBPM unit confirm a weak bunch defocusing effect in the vertical direction.

This observation, facilitated by removal of the exit slit, is shown in Figs. 3(a) and 3(b) where observed profiles with and without applied rf are compared. PARMILA also predicts this weak defocusing effect as seen in Figs. 3(c) and 3(d). The lineouts of the image data and the PARMILA predictions clearly show distributions with a broad base with rf on but with broad base combined with narrow central peak without rf. Furthermore with the exit slit reinstalled, PARMILA well predicts the transverse bunch profile of (FWHM) extent $20 \mathrm{~mm} \times 20 \mathrm{~mm}$ as can be seen in Fig. 4 when the rf is on.

A PARMILA simulation of the modulated spectrum exiting the rf phase rotation cavity and subsequently entering the monochromator is shown in Fig. 5(a). In this figure the observed peak centered at this energy represents the combined effects of the upstream PMQ triplet and the rf cavity energy compression at lower energy. The PMQ effect has

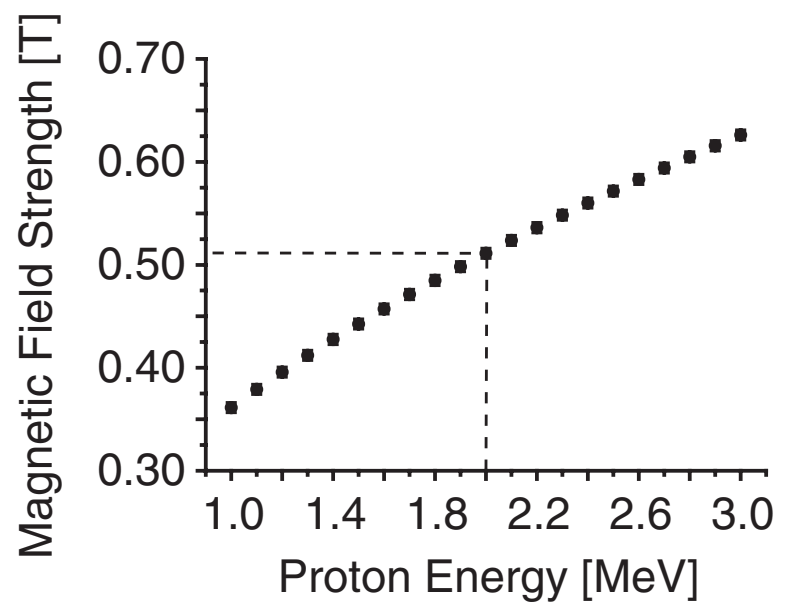

FIG. 6. Calibration curve of the monochromator, the energy of the transmitted proton beam through the slit vs the magnetic field strength of the bending magnet. been described in a previous report as the observation of a quasimonoenergetic proton spectrum with an energy peak near $2.4 \mathrm{MeV}$ and an increase in the number of $\sim 2.4 \mathrm{MeV}$ protons in the focal plane [13]. The spectral filtering imposed by the monochromator is characterized by the calibration shown in Fig. 6. The exit slit determines the 5\% energy spread at $1.9 \mathrm{MeV}$ which is confirmed in the PARMILA simulation result in Fig. 5(b). At the end of the transport line (plane " $\mathrm{g}$ " of Fig. 1 which is located $\sim 4 \mathrm{~m}$ from the source and $735 \mathrm{~mm}$ from plane "f"), we directly measure the transmitted bunch duration with the same TOF unit that was used to measure source spectra. At this location the peak current and bunch charge density are very low at $300 \mu \mathrm{A}$ and $2 \times 10^{5} \mathrm{~cm}^{-3}$, respectively. The solid line and open circles in Fig. 7(a) show typical experimental results measured at the plane "g" and the corresponding PARMILA simulation including the TOF response time. The slight discrepancy seen in the peak position of $\sim 1 \mathrm{~ns}$ and the shape at the right-hand side of the peak we attribute to the small uncertainty in the distance measurement to the detector ( $\sim 1 \mathrm{~ns}$ corresponds to $\sim 1 \mathrm{~cm}$ flight distance of the $2 \mathrm{MeV}$ proton beam) and the shot-to-shot fluctuations of the source parameters (the shot-to-shot fluctuation of the proton bunch charge has been measured to be $\sim 20 \%$ [13]). This is consistent with the 5\% transmitted energy spread predicted by PARMILA and indicates that there has been no observable modulation of the proton energy spectrum by the rf cavity at this energy. Figure 7(b) shows simulated baseline bunch duration of $6 \mathrm{~ns}$ without including the response time of the TOF unit (corresponding to an ultrafast TOF unit).

The simulated transmittance of the proton bunch through the entire transport line in the energy interval, $1.9 \pm 0.05 \mathrm{MeV}$ is $10 \%$ (which also corresponds to $0.3 \%$ for the total energy spectrum). Because the PMQs are not designed for the proton energy considered here, this transmittance can be improved with appropriate collection optics. We operate this beam line in a charge density and low peak current region for which space charge effects are insignificant [24]. 
(a)

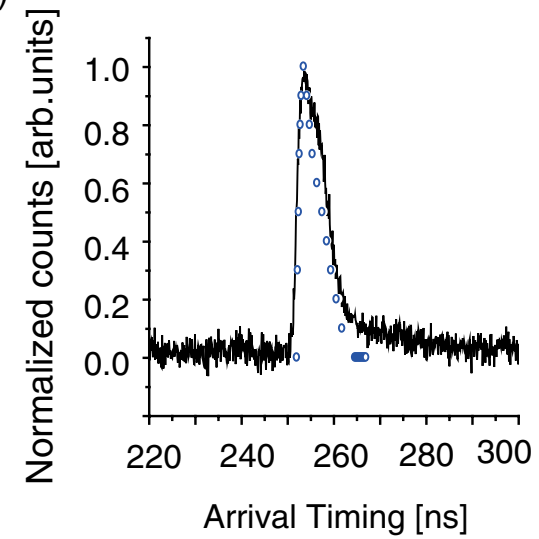

(b)

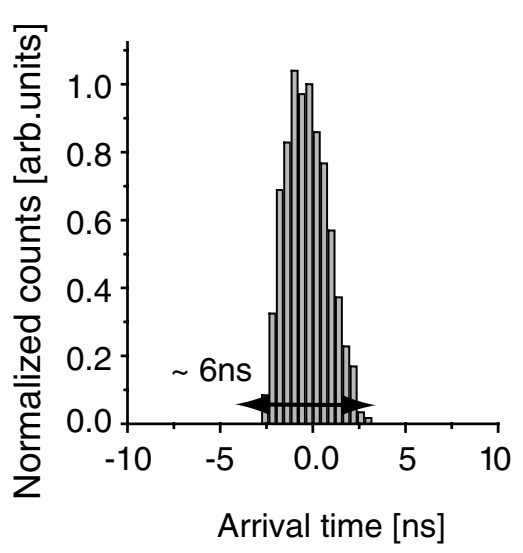

FIG. 7. (Color) (a) Normalized transmitted TOF signal measurement (black line) and the PARMILA simulation which includes the response time of the TOF spectrometer (open circles) at plane "g". (b) Simulated normalized transmitted TOF result which does not include the response time of the TOF spectrometer.

\section{CONCLUSION}

In conclusion, we have demonstrated, in the low space charge regime, $1 \mathrm{~Hz}$ operation of a low energy, quasimonoenergetic laser-driven test beam line $(1.9 \pm 0.05 \mathrm{MeV})$ that uses for the first time the combination of conventional PMQ's for charge collection, an rf cavity device, and a tunable monochromator. Weak defocusing by the rf cavity is observed with no observable alteration of the proton energy spectrum at this energy. It has also been shown that the well-established PARMILA code is reasonably predictive in the laser-driven case and can be considered useful for the design of integrated laser-driven ion accelerator systems in general. These optics tests and simulation comparisons can be pursued at increased proton energy, peak current, and repetition rate that would accompany further development of laser, proton source, and diagnostic capability. The effect of intrinsic comoving electrons, notably in close proximity to the source, can also be addressed in future simulations.

\section{ACKNOWLEDGMENTS}

We thank Dr. K. Nishio, Dr. T. Ishiyama, Dr. M. Katagiri, and Dr. K. Hasegawa for assisting us with ion measurement techniques and their many suggestions and useful discussions. We also gratefully acknowledge strong support from Dr. S. Kawanishi. This work has been financially supported by the Special Coordination Fund (SCF) for Promoting Science and Technology commissioned by the Ministry of Education, Culture, Sports, Science and Technology (MEXT) of Japan.

[1] R. A. Snavely, M. H. Key, S. P. Hatchett, T. E. Cowan, M. Roth, T.W. Phillips, M. A. Stoyer, E. A. Henry, T. C. Sangster, M. S. Singh, S.C. Wilks, A. MacKinnon, A. Offenberger, D. M. Pennington, K. Yasuike, A. B.
Langdon, B. F. Lasinski, J. Johnson, M. D. Perry, and E. M. Campbell, Phys. Rev. Lett. 85, 2945 (2000).

[2] S. C. Wilks, A. B. Langdon, T.E. Cowan, M. Roth, M. Singh, S. Hatchett, M.H. Key, D. Pennington, A. MacKinnon, and R.A. Snavely, Phys. Plasmas 8, 542 (2001).

[3] P. Mora, Phys. Rev. Lett. 90, 185002 (2003).

[4] M. Borghesi, A. J. Mackinnon, D. H. Campbell, D. G. Hicks, S. Kar, P. K. Patel, D. Price, L. Romagnani, A. Schiavi, and O. Willi, Phys. Rev. Lett. 92, 055003 (2004).

[5] T. E. Cowan, J. Fuchs, H. Ruhl, A. Kemp, P. Audebert, M. Roth, R. Stephens, I. Barton, A. Blazevic, E. Brambrink, J. Cobble, J. Fernandez, J.-C. Gauthier, M. Geissel, M. Hegelich, J. Kaae, S. Karsch, G. P. Le Sage, S. Letzring, M. Manclossi, S. Meyroneinc, A. Newkirk, H. Pepin, and N. Renard-LeGalloudec, Phys. Rev. Lett. 92, 204801 (2004).

[6] M. Nishiuchi, H. Daido, A. Sagisaka, K. Ogura, S. Orimo, K. Kado, A. Yogo, M. Mori, Y. Hayashi, S. Bulanov, A. Fukumi, Z. Li, A. Noda, and S. Nakamura, Appl. Phys. B 87, 615 (2007).

[7] K. Krushelnick, EL. Clark, R. Allott, F. Beg, C. N. Danson, A. Machacek, V. Malka, Z. Najmudin, D. Neely, P. A. Norreys, M. R. Salvati, M. I. K. Santala, M. Tatarakis, I. Watts, M. Zepf, A. E. Dangor, IEEE Trans. Plasma Sci. 28, 1184 (2000).

[8] A. Noda, S. Nakamura, Y. Iwashita, S. Sakabe, M. Hashida, T. Shirai, S. Shimizu, H. Tongu, H. Ito, H. Souda, A. Yamazaki, M. Tanabe, H. Daido, M. Mori, M. Kado, A. Sagisaka, K. Ogura, M. nishiuchi, S. Orimo, Y. Hayashi, A. Yogo, S. Bulanov, T. Esirkepov, A. Nagashima, T. Kimura, T. Tajima, T. Takeuchi, K. Matsukado, A. Fukumi, and Z. Li, Laser Phys. 16, 647 (2006).

[9] K. Harres, I. Alber, A. Tauschwitz, V. Bagnoud, H. Daido, M. Gunther, F. Nurnberg, A. Otten, M. Schollmeier, J. Schutrumpf, M. Tampo, and M. Roth, Phys. Plasmas 17, 023107 (2010).

[10] T. Esirkepov, M. Borghesi, S. V. Bulanov, G. Mourou, and T. Tajima, Phys. Rev. Lett. 92, 175003 (2004). 
[11] S. V. Bulanov and V.S. Khoroshkov, Plasma Phys. Rep. 28, 453 (2002).

[12] T. Tajima, J. Jpn. Soc. Therapeutic Radiat. Oncol. 9, 83 (1998).

[13] M. Nishiuchi, I. Daito, M. Ikegami, H. Daido, M. Mori, S. Orimo, K. Ogura, A. Sagisaka, A. Yogo, A. S. Pirozhkov, H. Sugiyama, H. Kiriyama, H. Okada, S. Kanazawa, S. Kondo, T. Shimomura, M. Tanoue, Y. Nakai, H. Sasao, D. Wakai, H. Sasaki, P. Bolton, I. W. Choi, J. H. Sung, J. Lee, Y. Oishi, T. Fujii, K. Nemoto, H. Souda, A. Nodai, Y. Iseki, and T. Yoshiyuki, Appl. Phys. Lett. 94, 061107 (2009).

[14] M. Schollmeier, S. Becker, M. Geissel, K. A. Flippo, A. Blazevic, S. A. Gaillard, D. C. Gautier, F. Gruner, K. Harres, M. Kimmel, F. Nurnberg, P. Rambo, U. Schramm, J. Schreiber, J. Schutrumpf, J. Schwarz, N. A. Tahir, B. Atherton, D. Habs, B. M. Hegelich, and M. Roth, Phys. Rev. Lett. 101, 055004 (2008).

[15] S. Ter-Avetisyan, M. Schnurer, R. Polster, P. V. Nickles, and W. Sandner, Laser Part. Beams 26, 637 (2008).

[16] H. Sakaki et al., in Proceedings of the 23rd Particle Accelerator Conference, Vancouver, Canada, 2009 (IEEE, Piscataway, NJ, 2009), TU6PFP009.

[17] H. Takeda, Parmila LANL Report No. LA-UR-98-4478, 2005.

[18] Robert W. Garnet, Status of the Los Alamos Accelerator Code Group http://laacg1.lanl.gov/laacg/laacg.pdf; S. Nath, J. Qiang, R. Ryne, J. Stovall, H. Takeda, L. Young, K. R. Crandall, N. Pichoff, and D. Uriot, in Proceedings of the 19th Particle Accelerator Conference, Chicago, Illinois, 2001 (IEEE, Piscataway, NJ, 2001), p. 264; M. A. Plum, Nucl. Phys. B154, 105 (2006).

[19] H. Kiriyama, M. Mori, Y. Nakai, T. Shimomura, M. Tanoue, A. Akutsu, H. Okada, T. Motomura, S. Kondo, S. Kanazawa, A. Sagisaka, J. Ma, I. Daito, H. Kotaki, H. Daido, S. V. Bulanov, T. Kimura, and T. Tajima, Opt. Lett. 33, 645 (2008).

[20] S. Nakamura, M. Ikegami, Y. Iwashita, T. Shirai, H. Tongu, H. Souda, H. Daido, M. Mori, M. Kado, A. Sagisaka, K. Ogura, M. Nishiuchi, S. Orimo, Y. Hayashi, A. Yogo, A.S. Pirozhkov, S. V. Bulanov, T.
Esirkepov, A. Nagashima, T. Kimura, T. Tajima, T. Tkeuchi, A. Fukumi, Z. Li, and A. Noda, Jpn. J. Appl. Phys. 46, L717 (2007); A. Wakita, Y. Iwashita, T. Shirai, M. Ikegami, H. Tongu, H. Souda, M. Mori, A. Yogo, S. Orimo, M. Nishiuchi, K. Ogura, A. Sagisaka, J.-L. M, A. S. Pirozhkov, H. Kiriyama, Y. Nakai, T. Shimomura, M. Tanoue, A. Akutsu, H. Okada, T. Motomura, S. Kondo, S. Kanazawa, H. Sugiyama, H. Daido, and A. Noda, Nucl. Instrum. Methods Phys. Res., Sect. A 599, 15 (2009).

[21] S. Nakamura, Y. Iwashita, A. Noda, T. Shirai, H. Tongu, A. Fukumi, M. Kado, A. Yogo, M. Mori, S. Orimo, K. Ogura, A. Sagisaka, M. Nishiuchi, Y. Hayashi, Z. Li, H. Daido, and Y. Wada, Jpn. J. Appl. Phys. 45, L913 (2006); A. Yogo, H. Daido, A. Fukumi, Z. Li, K. Ogura, A. Sagisaka, A. S. Pirozhkov, S. Nakamura, Y. Iwashita, T. Shirai, A. Noda, Y. Oishi, T. Nayuki, T. Fujii, K. Nemoto, I. W. Choi, J. H. Sung, D.-K. Ko, J. Lee, M. Kaneda, and A. Itoh, Phys. Plasmas 14, 043104 (2007).

[22] M. Ikegami, S. Nakamura, Y. Iwashita, T. Shirai, H. Souda, Y. Tajima, M. Tanabe, H. Tongu, H. Itoh, H. Shintaku, A. Yamazaki, H. Daido, A. Yogo, S. Orimo, M. Mori, M. Nishiuchi, K. Ogura, A. Sagisaka, A. S. Pirozhkov, H. Kiriyama, S. Kanazawa, S. Kondo, Y. Yamamoto, T. Shimomura, M. Tanoue, Y. Nakai, A. Akutsu, S. V. Bulanov, T. Kimura, Y. Oishi, K. Nemoto, T. Tajima, and A. Noda, Phys. Rev. ST Accel. Beams 12, 063501 (2009).

[23] L. Romagnani, J. Fuchs, M. Borghesi, P. Antici, P. Audebert, F. Ceccherini, T. Cowan, T. Grismayer, S. Kar, A. Macchi, P. Mora, G. Pretzler, A. Schiavi, T. Toncian, and O. Willi, Phys. Rev. Lett. 95195001 (2005).

[24] M. Antici, M. Fazi, A. Lombardi, M. Migliorati, L. Palumbo, P. Audebert, and J. Fuchs, J. Appl. Phys. 104, 124901 (2008).

[25] S. Fujimura and A. Ueno, in Proceedings of the 18th Particle Accelerator Conference, New York, 1999 (IEEE, New York, 1999), TUP10.

[26] M Borghesi, A Bigongiari, S. Kar, A. Macchi, L. Romagnani, P. Audebert, J. Fuchs, T. Toncian, O. Willi, S. V. Bulanov, A. J. Mackinnon, and J. C. Gauthier, Plasma Phys. Controlled Fusion 50124040 (2008). 